\title{
COMPOSTOS FENÓlICOS TOTAIS E ANTOCIANINAS EM SUCO DE UVA ${ }^{1}$
}

\author{
Cassia R. MALACRIDA², Silvana da MOTTA $^{2, *}$
}

\section{RESUMO}

As concentrações de compostos fenólicos totais e antocianinas em sucos de uva reconstituídos e simples, de diferentes marcas, disponíveis no comércio varejista da região metropolitana de Belo Horizonte (Minas Gerais) foram quantificadas. Também se determinou a contribuição das antocianinas poliméricas à cor e o índice de degradação das antocianinas. Os teores de compostos fenólicos totais variaram entre 0,27 e $2,41 \mathrm{~g} / \mathrm{L}$ e as concentrações de antocianinas de 1,17 a $66,80 \mathrm{mg} / \mathrm{L}$. Foram observadas diferenças significativas (Teste de Duncan, $\mathrm{p}<0,05$ ) nas concentrações de fenólicos totais e de antocianinas entre as diferentes marcas, tanto para o suco de uva reconstituído quanto para o simples. O percentual de contribuição das antocianinas poliméricas à cor também variou significativamente entre as marcas, sendo que os sucos de uva simples tiveram percentuais superiores $(81,6 \%)$ aos dos sucos de uva reconstituídos $(77,5 \%)$. Os sucos de uva reconstituídos apresentaram valor médio total de degradação das antocianinas $(3,92)$ maior que os sucos de uva simples $(3,44)$.

Palavras-chave: suco de uva, compostos fenólicos, antocianinas, antocianinas poliméricas, índice de degradação.

\section{SUMMARY}

TOTAL PHENOLICS AND ANTHOCYANINS IN GRAPE JUICE. Total phenolics and anthocyanins contents were quantified in simple and reprocessed grape juices of different brands, commercially available in Belo Horizonte (Minas Gerais, Brazil) metropolitan area. The contribution of polymeric anthocyanins to color and the anthocyanins degradation index were also determined. Total phenolics levels varied from 0,27 to $2,41 \mathrm{~g} / \mathrm{L}$ and anthocyanin concentrations from 1,17 to $66,80 \mathrm{mg} / \mathrm{L}$. Significant differences were found (Duncan's test, $\mathrm{p}<0,05$ ) in total phenolic and anthocyanin concentrations among different brands of reprocessed grape juice as well as the simple juice. The percent contribution of polymeric anthocyanins to color also varied significantly among different brands, the simple grape juice showing a higher percentage $(81,6 \%)$ than the reprocessed one $(77,5 \%)$. The latter presented total mean value of anthocyanins degradation $(3,92)$ higher than the former one $(3,44)$.

Keywords: grape juice, phenolic compounds, anthocyanins, polymeric anthocyanins, degradation index.

\section{1 - INTRODUÇÃO}

Os compostos fenólicos estão amplamente distribuídos no reino vegetal. São definidos como substâncias que possuem um anel aromático com um ou mais substituintes hidroxílicos, incluindo seus grupos funcionais [26]. Entre as frutas, a uva é uma das maiores fontes de compostos fenólicos. Os principais fenólicos presentes na uva são os flavonóides (antocianinas, flavanóis e flavonóis), os estilbenos (resveratrol), os ácidos fenólicos (derivados dos ácidos cinâmicos e benzóicos) e uma larga variedade de taninos [6].

As antocianinas são flavonóides que se encontram largamente distribuídos na natureza e são responsáveis pela maioria das cores azul, violeta e todas as tonalidades de vermelho que aparecem em flores, frutos, algumas folhas, caules e raízes de plantas [13, 33]. Nas videiras, elas acumulam-se nas folhas durante a senescência e são responsáveis pela coloração das cascas das uvas tintas, sendo encontradas também na polpa de algumas variedades de uvas [18].

O suco de uva é uma importante fonte de compostos fenólicos, no entanto, a quantidade e o tipo destes compostos não são necessariamente os mesmos da uva fresca. Os conteúdos de fenólicos totais e de antocianinas nas uvas variam de acordo com a espécie, variedade, maturidade, condições climáticas e cultivar [14, 26]. Determinados

${ }^{1}$ Recebido para publicação em 26/04/2004. Aceito para publicação em 06/09/2005 (001330).

${ }^{2}$ Universidade Federal de Minas Gerais - Departamento de Alimentos, Faculdade de Farmácia, Av. Antônio Carlos no 6627, CEP 31270-010, Belo Horizonte-MG. E-mail:smotta@ciclope.lcc.ufmg.br

* Para quem a correspondência deve ser enviada. tratamentos aos quais a uva e o mosto são submetidos durante a produção do suco tais como tipo de extração, tempo de contato entre o suco e as partes sólidas da uva (casca e sementes), prensagem, tratamentos térmicos, tratamentos enzimáticos e adição de dióxido de enxofre e ácido tartárico também interferem na quantidade destes compostos no suco pronto [7, 26, 29].

A extração a quente contribui para uma maior concentração de fenólicos no suco [3, 7]. Entretanto, o uso de altas temperaturas durante a extração, pasteurização e estocagem pode acarretar perdas na quantidade de compostos fenólicos, principalmente devido à degradação de antocianinas [4, 12].

Durante o armazenamento podem ocorrer mudanças no aroma, cor e sabor do suco devido à redução na concentração de antocianinas monoméricas e formação de pigmentos poliméricos. As reações responsáveis por essas transformações incluem, freqüentemente, a condensação direta entre antocianinas e flavonóis e a polimerização das próprias antocianinas [5].

Estudos epidemiológicos têm demonstrado a associação entre o consumo de alimentos e bebidas ricos em compostos fenólicos e a prevenção de doenças, tais com câncer [30] e doenças coronarianas isquêmicas (DCI) [18].

Muitas pesquisas têm sido realizadas avaliando os efeitos antioxidantes dos compostos fenólicos presentes no vinho [26], entretanto, alguns autores verificaram, em sucos de uva, atividade antioxidante similar à encontrada em vinhos tintos [7, 33]. O suco de uva contém mais compostos fenólicos gli- 
cosilados do que o vinho [28], sendo que, segundo HOLLMAN et al. [11], estes compostos são mais facilmente absorvidos pelo organismo do que suas respectivas agliconas. Por outro lado, a presença de etanol no vinho aumenta a absorção de fenólicos, pois previne a precipitação de polifenóis no trato digestivo [25]. Entretanto, existem estudos que contradizem este possível efeito benéfico do consumo combinado de álcool e compostos fenólicos. VINSON et al. [34] comparou a eficiência do vinho tinto, vinho tinto sem álcool e suco de uva sobre a aterosclerose. As concentrações de polifenóis das três bebidas foram normalizadas a um mesmo valor para o teste com os animais. A comparação dos resultados obtidos revelou que o suco de uva foi duas vezes mais efetivo na diminuição do colesterol do que o vinho tinto e seu equivalente sem álcool e duas vezes mais eficiente que o vinho tinto com relação à inibição da aterosclerose. Comparando-se o vinho tinto e o vinho tinto sem álcool verificou-se que os efeitos benéficos das duas bebidas foram praticamente os mesmos, o que demonstra que o etanol não representa benefícios adicionais ao vinho.

As propriedades biológicas dos polifenóis dependem também da sua biodisponibilidade. Estudos de biodisponibilidade de compostos fenólicos determinam a concentração no plasma e na urina após a ingestão destes compostos. Concentrações muito baixas da ordem de $\mu \mathrm{M}$ foram detectadas no plasma e a maior parte dos polifenóis ingeridos não foi encontrada na urina, isto sugere que os compostos não foram absorvidos pela membrana da parede intestinal, ou foram absorvidos e excretados na bile ou metabolizados pela microflora intestinal ou nos tecidos [11, 24]

O consumo de suco de uva como fonte de compostos fenólicos pode apresentar vantagem com relação ao do vinho, já que a ausência de álcool permite que o suco seja consumido pela maioria das pessoas, inclusive aquelas portadoras de algumas doenças, por exemplo, a hepatite, e crianças [22]. Além disso, o vinho pode conter substâncias, tais como uréia, metanol e aminas biogênicas, que quando consumidas indiscriminadamente podem causar efeitos tóxicos no organismo humano $[9,10,16]$.

Este trabalho teve como objetivo determinar o conteúdo de compostos fenólicos totais, a concentração de antocianinas, o percentual de contribuição das antocianinas poliméricas à cor e o índice de degradação das antocianinas em sucos de uva tintos reconstituídos e simples, de diferentes marcas, disponíveis no comércio varejista da região metropolitana de Belo Horizonte-MG.

\section{2 - MATERIAL E MÉTODOS}

\section{1 - Material}

\subsection{1 - Amostras}

Para as análises foram utilizadas 8 marcas de sucos de uva tintos reconstituídos e 12 marcas de diferentes sucos de uva tintos simples. Foram analisadas três embalagens do mesmo lote de cada marca de suco, sendo cada embalagem analisada em duplicata. Em nenhuma das marcas constava no rótulo informações sobre a variedade da uva utilizada na elaboração do suco e a safra vitícola.

\section{2 - Métodos de análise}

\subsection{1 - Fenólicos totais}

O conteúdo de compostos fenólicos totais foi determinado segundo metodologia descrita por SWAIN \& HILLIS [31], utilizando-se espectrofotômetro UV-Visível 160A da Shimadzu (Kyoto, Japão) para as medidas de absorbâncias das amostras.

\subsection{2 - Antocianinas monoméricas}

O conteúdo de antocianinas monoméricas foi determinado pelo método do $\mathrm{pH}$ diferencial descrito por WROLSTAD [35], utilizando-se espectrofotômetro UV-Visível 160A da Shimadzu (Kyoto, Japão) para as medidas de absorbâncias das amostras. O conteúdo de antocianinas monoméricas no suco de uva foi calculado como malvidina-3,5-diglicosídio.

\subsection{3 - Índice de degradação das antocianinas}

Segundo metodologia descrita por FULEKI \& FRANCIS [8], o índice de degradação das antocianinas é calculado dividindo-se o total de antocianinas calculado pelo método do $\mathrm{pH}$ único pelo total de antocianinas calculado pelo método do $\mathrm{pH}$ diferencial.

\subsection{4 - Contribuição das antocianinas poliméricas à cor}

O percentual de contribuição de antocianinas poliméricas à cor foi determinado segundo metodologia descrita por WROLSTAD [35], utilizando-se espectrofotômetro UVVisível 160A da Shimadzu (Kyoto, Japão) para as medidas de absorbâncias das amostras.

\section{3 - Análise estatística}

Os resultados dos experimentos foram tabulados e submetidos à análise de variância, utilizando-se delineamento inteiramente casualizado. As médias foram comparadas pelo Teste de Duncan a 5\% de probabilidade [17]. Os coeficientes de correlação entre as determinações analíticas foram obtidos com auxílio do programa estatístico Statistica versão 5.1 (StatSoft Inc., Tulsa, EUA) e a significância destes coeficientes foi verificada pelo Teste $t$ [23].

\section{3 - RESULTADOS E DISCUSSÃO}

Os teores de compostos fenólicos totais variaram entre 0,27 e 1,32 g/L nos sucos de uva reconstituídos (Tabela 1), e entre 0,60 e 2,41 g/L nos sucos de uva simples (Tabela 2). A marca $\mathrm{E}$ apresentou as maiores concentrações de fenólicos totais entre as marcas de sucos reconstituídos (1,32 g/L) e entre as de sucos simples $(2,41 \mathrm{~g} / \mathrm{L})$. 
TABELA 1 - Teores médios de compostos fenólicos totais, antocianinas monoméricas, contribuição das antocianinas poliméricas à cor e índice de degradação das antocianinas em sucos de uva reconstituídos

\begin{tabular}{|c|c|c|c|c|}
\hline Marcas & $\begin{array}{c}\text { Compostos Fenólicos } \\
\text { Totais }(\mathbf{g} / \mathbf{L})^{*}\end{array}$ & $\begin{array}{l}\text { Antocianinas monoméricas } \\
(\mathrm{mg} / \mathrm{L})^{*}\end{array}$ & $\begin{array}{l}\text { Contribuição das antocianinas } \\
\text { poliméricas à cor }(\%)^{*}\end{array}$ & Índice de degradação* \\
\hline A & $1,29^{\mathrm{a}} \pm 0,16$ & $36,23^{a} \pm 0,94$ & $78,7^{\mathrm{b}} \pm 4,7$ & $2,03^{b} \pm 0,05$ \\
\hline B & $0,86^{c} \pm 0,07$ & $16,94^{\mathrm{d}} \pm 0,79$ & $68,4^{\circ} \pm 4,6$ & $2,42^{b} \pm 0,04$ \\
\hline C & $0,27^{d} \pm 0,01$ & $2,13^{f} \pm 0,66$ & $88,9^{a} \pm 2,6$ & $12,38^{a} \pm 4,42$ \\
\hline D & $1,17^{b} \pm 0,03$ & $22,02^{b} \pm 1,50$ & $84,8^{a} \pm 1,7$ & $3,00^{\mathrm{b}} \pm 015$ \\
\hline G & $1,05^{b} \pm 0,04$ & $16,26^{d} \pm 0,65$ & $70,6^{c} \pm 2,1$ & $2,74^{b} \pm 0,06$ \\
\hline $\mathrm{H}$ & $1,10^{\mathrm{b}} \pm 0,01$ & $19,37^{\circ} \pm 1,00$ & $78,4^{\mathrm{b}} \pm 1,7$ & $3,02^{b} \pm 0,09$ \\
\hline Média total & $1,05 \pm 0,07$ & $17,31 \pm 1,90$ & $77,5 \pm 1,5$ & $3,92 \pm 0,72$ \\
\hline
\end{tabular}

*Valores médios entre triplicatas \pm desvio padrão com letras diferentes na mesma coluna indicam diferenças significativas entre as marcas (Teste de Duncan, p<0,05)

TABELA 2 - Teores médios de compostos fenólicos totais, antocianinas monoméricas, contribuição das antocianinas poliméricas à cor e índice de degradação das antocianinas em sucos de uva simples

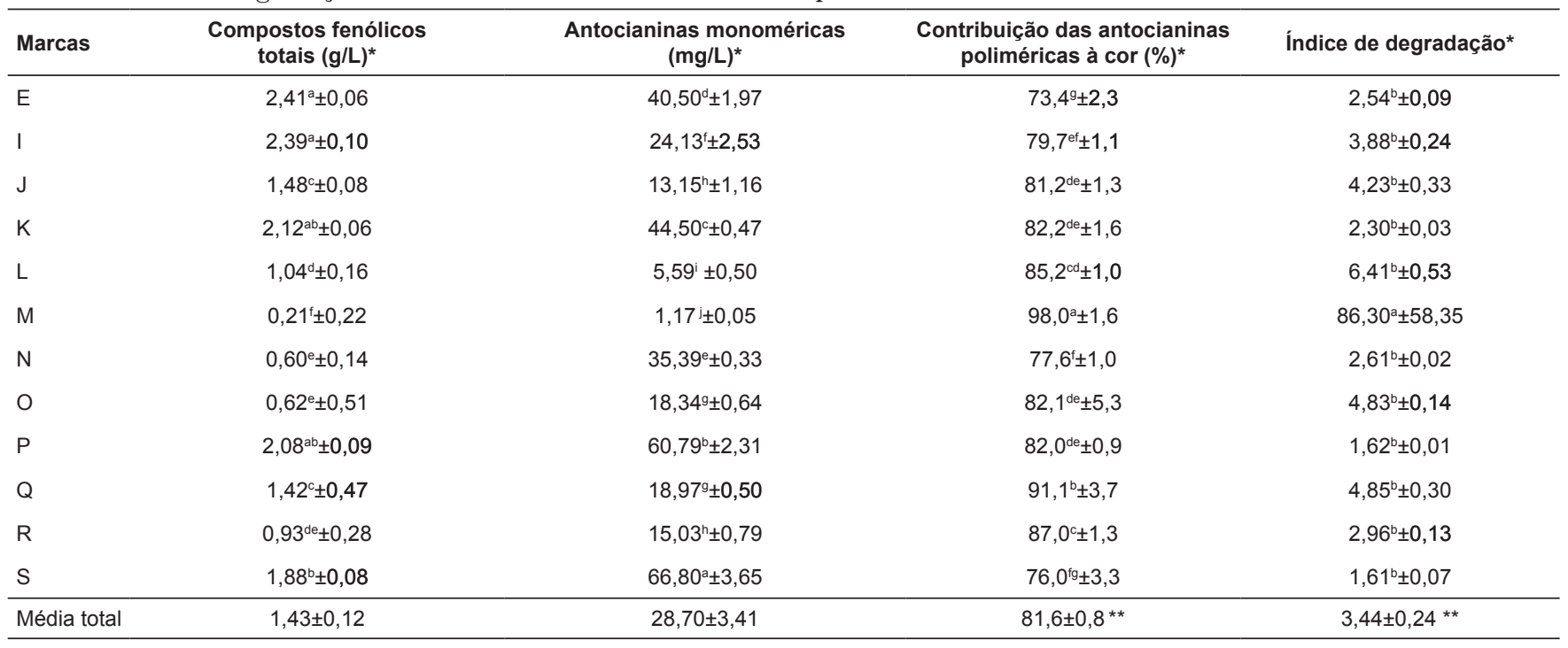

*Valores médios entre triplicatas \pm desvio padrão com letras diferentes na mesma coluna indicam diferenças significativas entre as marcas (Teste de Duncan, $\mathrm{p}<0,05$ ) **Média total calculada entre as marcas excluindo-se a marca M

Observou-se diferença significativa $(\mathrm{p}<0,05)$ no conteúdo de fenólicos totais entre algumas marcas tanto no suco de uva simples quanto no reconstituído. Tal fato indica a influência de possíveis diferenças de processamento (tipo e tempo de extração, tratamento térmico, tratamento enzimático, etc) entre as marcas sobre o parâmetro analisado.

A variedade de uva utilizada no processamento do suco também pode ser uma causa de variação nos teores de compostos fenólicos entre as marcas. O conteúdo de fenólicos no suco varia de acordo com a variedade da uva, maturidade e regiões e práticas de cultivo das mesmas. Nenhuma das marcas analisadas continha informações no rótulo sobre a variedade, safra ou região produtora da uva utilizada na fabricação do suco.

Os sucos de uva simples apresentaram um valor médio de fenólicos totais $(1,43 \mathrm{~g} / \mathrm{L})$ superior ao dos sucos de uva reconstituídos (1,05 g/L). As etapas de processamento do suco de uva reconstituído diferem das do suco simples, já que o primeiro é produzido a partir de diluição de suco de uva concentrado [1]. Durante a concentração do suco podem ocorrer perdas no conteúdo de compostos fenólicos devido, principalmente, às temperaturas elevadas que o suco é submetido durante a recuperação de aromas e a evaporação propriamente dita. A concentração de fenólicos no suco de uva é influenciada pelo tipo de extração e procedimentos empregados na produção do suco e pelas reações que ocorrem durante o seu armazenamento [7, 12].

FRANKEL et al. [7] determinaram a concentração de fenólicos totais em sucos de uva comerciais produzidos a partir de uvas da variedade Concord e de misturas de diferentes variedades de uvas. O método utilizado para a quantificação dos fenólicos totais foi o de Folin-Ciocalteu e o padrão foi o ácido gálico. As médias obtidas foram de 
$1,79 \mathrm{~g} / \mathrm{L}$ para os sucos de uva Concord e de $1,47 \mathrm{~g} / \mathrm{L}$ para aqueles produzidos a partir de misturas de diferentes variedades de uvas. Os sucos de uva simples analisados no presente trabalho apresentaram uma média de fenólicos totais de $1,43 \mathrm{~g} / \mathrm{L}$, valor este próximo ao encontrado para os sucos elaborados com diferentes variedades de uva.

Devido à escassez de dados na literatura referentes ao conteúdo de compostos fenólicos totais e de antocianinas em suco de uva, os resultados obtidos no presente trabalho foram comparados aos encontrados por vários autores para o vinho tinto.

Os conteúdos de fenólicos totais encontrados para os sucos de uva reconstituídos (0,27-1,32 g/L) e sucos de uva simples $(0,60-2,41 \mathrm{~g} / \mathrm{L})$ analisados foram semelhantes aos encontrados na literatura para o vinho tinto. Em vinhos italianos das uvas Merlot, Cabernet Sauvignon, Cabernet Franc, Sangiovese, Gamay entre outras, foram relatados valores que variaram de 0,33 a 1,60 g/L em vinhos jovens e 0,53 a 2,05 g/L em vinhos envelhecidos [15]. TORRES [32] relatou valores médios de fenólicos totais de $1,41 \mathrm{~g} / \mathrm{L}$ em vinhos tintos Merlot e de 1,34 g/L em vinhos tintos Cabernet Franc produzidos na região sul do Brasil, safra 1999.

A concentração média de antocianinas nos sucos de uva reconstituídos variou de 2,13 a 36,23 mg/L (Tabela 1) e de 1,17 a $66,80 \mathrm{mg} / \mathrm{L}$ (Tabela 2 ) nos sucos de uva simples. Verificou-se diferença significativa $(p<0,05)$ entre algumas marcas tanto nos sucos reconstituídos quanto nos simples. Cultivar, maturidade, ano de produção e outros fatores ambientais afetam o conteúdo de antocianinas das uvas e conseqüentemente do suco de uva [14].

As principais variedades de uvas utilizadas na produção de suco no Brasil são Concord, Isabel, Bordô e Jacquez, sendo que as duas últimas são as mais ricas em pigmentos antociânicos [20, 21]. Possivelmente, os sucos que apresentaram teores médios elevados de antocianinas (marcas $\mathrm{E}, \mathrm{K}, \mathrm{P}$ e S dos sucos de uva simples e marca A dos sucos de uva reconstituídos) correspondem a sucos processados a partir de uvas Bordô e Jacquez.

As diferenças de concentrações entre as diversas marcas de suco também podem ser explicadas por diferenças no processamento dos mesmos, principalmente no tipo e tempo de extração, tratamento térmico, tratamentos enzimáticos e condições de estocagem [12, 29].

Os sucos de uva simples apresentaram uma concentração média de antocianinas $(28,70 \mathrm{mg} / \mathrm{L})$ superior à dos sucos de uva reconstituídos (17,31 g/L). Estas concentrações foram similares às citadas na literatura para vinhos. BURNS et al. [2] avaliaram a concentração de antocianinas em vinhos tintos Cabernet Sauvignon e Merlot de diversas safras e de vários países incluindo Chile, Estados Unidos, França, Espanha e Bulgária. As concentrações encontradas em vinhos Cabernet Sauvignon variaram de 11,00 a 32,57 mg/L, e em vinhos Merlot, variaram de 19,11 a 23,44mg/L. TORRES [32] determinou os teores de antocianinas em vinhos tintos Merlot e Cabernet Franc produzidos na região sul do Brasil, safra 1999. Os valores médios verificados foram de 28,39 mg/L para os vinhos Merlot e de 20,41 mg/L para os vinhos Cabernet Franc.

Os percentuais médios de contribuição das antocianinas poliméricas à cor em sucos de uva reconstituídos variaram de 68,4 a $88,9 \%$ (Tabela 1 ) e em sucos de uvas simples variaram de 73,4 a 91,1\% (Tabela 2). Constataram-se, entre as marcas, diferenças significativas $(p<0,05)$ nestes percentuais para os dois tipos de sucos, sendo que os sucos de uva simples apresentaram maior percentual médio $(81,6 \%)$ que os sucos de uva reconstituídos $(77,5 \%)$. No cálculo da média total de contribuição das antocianinas poliméricas à cor no suco simples, o valor médio da marca M foi excluído uma vez que esta marca apresentou coloração amarelada, não característica de suco de uva.

Durante as etapas de processamento e, principalmente, durante o armazenamento do suco, o conteúdo de antocianinas monoméricas decresce progressiva e irreversivelmente formando pigmentos poliméricos mais estáveis. Estes pigmentos são responsáveis por mudanças no aroma, cor e sabor do suco [5].

Nos sucos de uva reconstituídos a marca C apresentou o menor valor médio de antocianinas $(2,13 \mathrm{mg} / \mathrm{L})$ e o maior percentual de contribuição de antocianinas poliméricas à cor $(88,9 \%)$. Fato similar foi verificado nos sucos de uvas simples onde a marca $\mathrm{M}$ com menor valor médio de antocianinas (1,2 mg/L) também apresentou o maior percentual de contribuição de antocianinas poliméricas à cor $(98,0 \%)$.

Com relação ao índice de degradação das antocianinas, os sucos de uva reconstituídos apresentaram valor médio total $(3,92)$ maior que os sucos de uva simples $(3,44)$. De maneira análoga à contribuição das antocianinas poliméricas à cor, a média total do índice de degradação do suco simples foi calculada excluindo-se a marca M. Além de não apresentar coloração característica de suco de uva, nesta marca verificou-se concentrações muito baixas de compostos fenólicos $(0,21 \mathrm{~g} / \mathrm{L})$ e antocianinas monoméricas $(1,17 \mathrm{mg} / \mathrm{L})$ e um índice de degradação muito superior às demais marcas $(86,30)$. Tal fato leva a crer que a degradação ocorrida nesta marca foi decorrente de alguma falha no processamento do lote analisado.

O aquecimento durante o processamento e a estocagem é uma das principais causas de degradação das antocianinas. O suco reconstituído é obtido pela dissolução do suco simples concentrado. Após a dissolução, o suco reconstituído é pasteurizado ou esterilizado dependendo do tipo de envase [13]. Desta forma, o suco reconstituído é submetido a mais tratamentos térmicos que o suco simples, podendo esta ser a explicação da maior degradação de antocianinas que ocorre neste tipo de suco.

Os coeficientes de correlação entre as concentrações de compostos fenólicos totais, antocianinas monoméricas, percentagem de contribuição das antocianinas poliméricas à cor e índice de degradação das antocianinas estão indicados na Tabela 3. 
TABELA 3 - Coeficientes de correlação entre fenólicos totais (CFT), antocianinas monoméricas (ANT), contribuição das antocianinas poliméricas à cor (CP) e índice de degradação (ID) das antocianinas nos sucos de uva reconstituído e simples

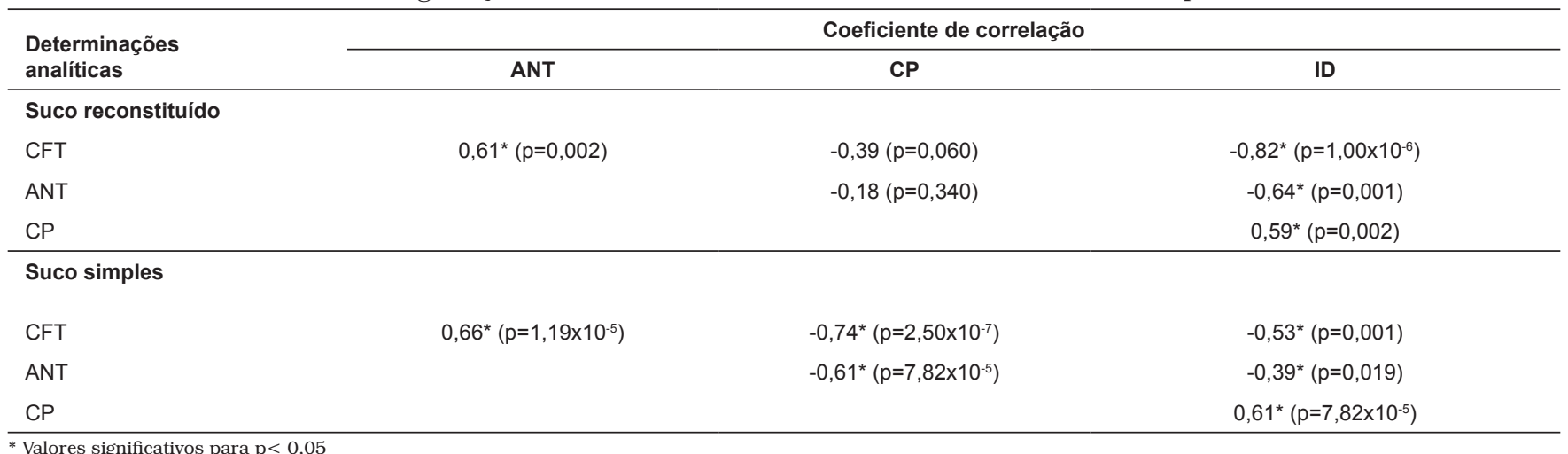

Todos os parâmetros analisados correlacionaram-se significativamente $(\mathrm{p}<0,05)$, sendo que somente nos sucos reconstituídos a percentagem de contribuição das antocianinas poliméricas à cor não se correlacionou com as concentrações de antocianinas monoméricas e compostos fenólicos totais.

Os fenólicos totais e as antocianinas monoméricas apresentaram coeficientes de correlação positivos de 0,61 e 0,66 nos sucos reconstituídos e simples, respectivamente. Tal fato indica a tendência entre os sucos analisados de que altas concentrações de fenólicos totais são acompanhadas de elevadas concentrações de antocianinas monoméricas.

A percentagem de contribuição das antocianinas poliméricas à cor apresentou correlações negativas com as concentrações de fenólicos totais e antocianinas monoméricas, porém, estas correlações só foram significativas nos sucos simples. A polimerização das antocianinas monoméricas provoca uma diminuição na concentração destas antocianinas no suco e, conseqüentemente, uma redução nos teores de compostos fenólicos totais.

O índice de degradação das antocianinas correlacionouse negativamente com as concentrações de fenólicos totais e antocianinas monoméricas nos dois tipos de sucos. Os coeficientes de correlação com os compostos fenólicos totais foram de -0,82 e -0,53, e com as antocianinas monoméricas foram de -0,64 e -0,39 para os sucos reconstituídos e simples, respectivamente.

Com relação à contribuição das antocianinas poliméricas à cor e o índice de degradação das antocianinas, foi verificada correlação positiva significativa tanto nos sucos reconstituídos $(0,59)$ quanto nos simples $(0,61)$. Tal fato indica que a polimerização é uma das principais causas de degradação das antocianinas no suco de uva, já que quanto maior a contribuição das antocianinas poliméricas à cor, maior o valor deste índice.

\section{4 - CONCLUSÕES}

- As concentrações de fenólicos totais, antocianinas monoméricas e o percentual de contribuição das antocianinas poliméricas à cor variaram significativamente entre as marcas tanto para o suco de uva simples quanto para o reconstituído;

- Os conteúdos de fenólicos totais encontrados para os sucos de uva reconstituídos e sucos de uva simples analisados foram semelhantes aos encontrados por vários autores para o vinho tinto. Assim, o suco de uva pode ser considerado uma boa fonte de compostos fenólicos;

- As marcas de suco com as menores concentrações de antocianinas monoméricas apresentaram elevado percentual de contribuição de antocianinas poliméricas à cor e os maiores índices de degradação das antocianinas;

- Os sucos de uva reconstituídos apresentaram valor médio total de degradação das antocianinas maior que os sucos de uva simples.

\section{5 - REFERÊNCIAS BIBLIOGRÁFICAS}

[1] BRASIL. Decreto n. 2314 de 04 de setembro de 1997. Regulamenta a Lei n. 8.918 de 14 de julho de 1994 que dispõe sobre a padronização, a classificação, o registro, a inspeção, a produção e a fiscalização de bebidas. Diário Oficial da República Federativa do Brasil, Brasília, DF, 05 set. 1997. Disponível em: <http://www.e-legis.bvs.br/leisref/public/showAct. php?id=619 > . Acesso em: 24 maio 2002.

[2] BURNS, J.; GARDNER, P.T.; O’NEIL, J.; CRAWFORD, S.; MORECROFT, I.; MC PHAIL, D.B.; LISTER, C.; MATHEWS, D.; MC LEAN, M.R.; LEAN, M.E.J.; DUTHIE, G.G.; CROZIER, A. Relationship among antioxidant activity, vasodilatation capacity, and phenolics content of red wines. J. Agric. Food Chem., v. 48, p. 220-230, 2000.

[3] CREASY, L.L.; CREASY, M.T. Grape chemistry and the significance of resveratrol: an overview. Pharm. Biol., v. 36, p. 8-13, 1998.

[4] DERGAL, S.B. Química de los Alimentos. $3^{\text {a }}$ ed. Nancalpon de Juárez: Longman de México, 1993, p. 388-397.

[5] FRANCIA-ARICHA, F.M.; GUERRA, M.T.; RIVAS-GONZALO, I.C.; SANTOS-BUELGA, C. New anthocyanin 
pigments formed after condensation with flavonols. J. Agric. Food Chem., v. 45, p. 2262-2266, 1997.

[6] FRANCIS, F.J. Anthocyanins and betalains: composition and applications. Cereal Foods World, v. 45, p. 208-213, 2000.

[7] FRANKEL, E.N.; BOSANEK, C.A.; MEYER, A.S.; SILLIMAN, K.; KIRK, L.L. Commercial grape juice inhibits the in vitro oxidation of human low-density lipoproteins. J. Agric. Food Chem., v. 46, p. 834-838, 1998.

[8] FULEKI, T.; FRANCIS, F.J. Quantitative methods for anthocyanins. 2. Determination of total anthocyanin and degradation index for cranberry juice. J. Food Sci., v. 33, p. 78-83, 1968.

[9] GLÓRIA, M.B.A.; WATSON, B.T.; SIMON-SARKADI, L.; DAESCHEL, M.A. A survey of biogenic amines in Oregon Pinot Noir and Cabernet Sauvignon wines. Am. J. Enol. Vitic., v. 49, p. 279-282, 1998.

[10] HASHIZUME, T.; MORETTI, R.H. Estudo de álcool metílico em vinhos nacionais. Col. Ital, v. 2, p. 135159, 1967/68.

[11] HOLLMAN, P.C.H.; VRIES, J.H.M.; VAN LEEUWEN, S.D.; MENGELERS, M.J.B.; KATAN, M.B. Absorption of dietary quercetin glycosides and quercetin in healthy ileostomy volunteers. Am. J. Clin. Nutr., v. 62, p. 1276-1282, 1995.

[12] JACKMAN, R.L.; SMITH, J.L. Anthocyanins and betalains. In: HENDRY, G.A.F.; HOUGHTON, J.D. (Eds.) Natural Food Colorants. $2^{\text {nd }}$ ed. Londres: Chapman \& Hall, 1996, p. 245-309.

[13] MARKAKIS, P. Stability of anthocyanins in foods. In: MARKAKIS, P. (Ed.) Anthocyanins as Food Colors. New York: Academic Press, 1982, p. 163-180.

[14] MAZZA, G. Anthocyanins in grapes and grape products. Crit. Rev. Food Sci. Nut., v. 35, p. 341-371, 1995.

[15] PELLEGRINI, N.; SIMONETTI, P.; GARDANA, C.; BRENNA, O.; BRIGHENTI, F.; PIETTA, G. Polyphenol content and total activity of Vini Novelli (young red wines). J. Agric. Food Chem., v. 48, p. 732-735, 2000.

[16] PEREIRA, C.N.; DAUDT, C.E. Uréia: sua determinação e presença em vinhos brasileiros. Cienc. Tecnol. Aliment., v. 15, p. 40-42, 1995.

[17] PIMENTEL-GOMES, F. Curso de Estatistica Experimental. $14^{\mathrm{a}}$ ed. Piracicaba: Nobel, 2000, 477 p.

[18] RENAUD, S.; DE LORGERIL, M. Wine, alcohol, platelets, and french paradox for coronary heart disease. Lancet, v. 339, p. 1523-1526, 1992

[19] REVILLA, E.; RYAN, J.M.; MARTIN-ORTEGA, G. Comparison of several procedures used for the extraction of anthocyanins from red grape. J. Agric. Food Chem., v. 46, p. 4592-4597, 1998.

[20] RIZZON, L.A.; MIELE, A. Características analíticas de sucos de uva elaborados no Rio Grande do Sul. Bol. sbCTA, v. 29, p. 129-133, 1995.

[21] RIZZON, L.A.; MANFROI, V.; MENEGUZZO, J. Elaboração de Suco de Uva na Propriedade Vitícola. Bento Gonçalves: Embrapa Uva e Vinho, 1998, 24 p.
[22] ROMERO-PÉREZ, A.I.; IBERN-GÓMES, M.; LAMUELARAVENTÓS, R.M.; TORRE-BORONAT, M.C. Piceid, the major resveratrol derivative in grape juice. J. Agric. Food Chem., v. 47, p. 1533-1536, 1999.

[23] SAMPAIO, I.B.M. Estatística Aplicada à Experimentação Animal. Belo Horizonte: Fundação de Ensino e Pesquisa em Medicina Veterinária e Zootecnia, 1998, $211 \mathrm{p}$.

[24] SCALBERT, A.; WILLIAMSON, G. Dietary intake and bioavailability of polyphenols. J. Nutri., v. 130, p. 2073-2085, 2000.

[25] SERAFINI, M.; MAIANI, G.; FERRO-LUZZI, A.F. Effect of ethanol on red wine tannin-protein (BSA) interactions. J. Agric. Food Chem., v. 45, p. 3148-3152, 1997.

[26] SHAHIDI, F.; NACZK, M. Food Phenolics: sources, chemistry, effects and applications. Lancaster: Technomic, 1995, $331 \mathrm{p}$.

[27] SHRINKHANDE, A.J. Wine by-products with health benefits. Food Res. Int., v. 31, p. 469-474, 2000.

[28] SINGLETON, V.L. Oxygen with phenols and related reactions in musts, wines and model systems: observations and practical implications. Am. J. Enol. Vitic., v. 38, p. 69-77, 1987.

[29] SISTRUNK, W.A.; GASCOIGNE, H.L. Stability of color in Concord grape juice and expression of color. J. Food Sci., v. 48, p. 430-435, 1983.

[30] STEINMETZ, K.A.; POTTER, J.D. Vegetables, fruit, and cancer prevention: a review. J. Am. Diet. Assoc., v. 54, p. 1027-1039, 1996

[31] SWAIN, T.; HILLIS, W.E. The phenolic constituents of prunus domestica. The quantitative analysis of phenolic constituents. J. Sci. Food Agric., v. 10, p. 63-68, 1959.

[32] TORRES, A.G. Avaliação de Compostos Fenólicos em Vinhos Tintos Brasileiros Cabernet Sauvignon, Cabernet Franc e Merlot. Belo Horizonte, 2002, 107 f. Dissertação (Mestre em Ciência de Alimentos), Faculdade de Farmácia, Universidade Federal de Minas Gerais (UFMG).

[33] VINSON, J.A.; JANG, J.; YANG, J.; DABBAGH, Y.; LIANG, X.; SERRY, M.; PROCH, J.; CAI, S. Vitamins and especially flavonoids in common beverages are powerful in vitro antioxidants which enrich lower density lipoproteins and increase their oxidative resistance after ex vivo spiking in human plasma. J. Agric. Food Chem., v. 47, p. 2502-2504, 1999.

[34] VINSON, J.A.; TEUFEL, K.; WU, N. Red wine, dealcoholized red wine, and especially grape juice, inhibit atherosclerosis in a hamster model. Atherosclerosis, v. 156, p. 67-72, 2001.

[35] WROLSTAD, R.E. Colors and Pigment Analysis in Fruit Products. Corvallis: Oregon Agricultural Experimental Station, 1976, 17 p.

\section{6 - AGRADECIMENTOS}

A CAPES, pelo apoio financeiro. 